\title{
Histopathological Study of Benzene Exhaust Emission Effect on Organs of White Rats
}

\author{
H. A. Naji \\ Coll. Vet. Med. University Al-Qadisiya
}

\begin{abstract}
The study designed on (20) white rats, the animals divided into (2) groups. The first group contain (10) rats act as control group, the second group contain (10) rats act as treated group, for (24) days continuously. The animals received for 1/4 hours per day to the benzene exhaust emissions which result from motor vehicle. The study showed that benzene emission cause damage and severs toxicity in tissue of lung, heart, liver, spleen, kidney and testis.
\end{abstract}

\section{Introduction}

Petroleum are largest chemical industries that are responsible for the emission of several pollutants into the atmosphere, benzene is one of commonly compounds found in the environment, industrial processes are the main sources of benzene in the environment(1). Benzene levels in the air can be elevated by emissions from burning coal and oil, benzene waste and storage operations, motor vehicle exhaust, and evaporation from gasoline service stations, tobacco smoke is another source of benzene in air, particularly indoors. Industrial discharge, disposal of products containing benzene, and gasoline leaks from underground storage tanks release benzene into water and soil (2). Benzene exhaust emission is a mixture of particles and gases, it contains more than several hundred different organic and inorganic components, including many chemicals that have been designated as toxic air pollutant (3). Sulfur dioxide (SO2), nitrogen dioxide (NO2) ,carbon monoxide (CO) and ozone (O3) these mixture gases result from benzene

\section{Materials and Methods}

The present study was conducted on (20) white rats and body weight $(250 \pm 5)$ .The animals were housed in $(6 \times 4 \times 3) \mathrm{m}^{3}$ room in animal house under 12 hours light / 12 hours dark at $(28 \pm 2)^{\circ}$ c. This study continued for (24) days.

Treatment:- Benzene exhaust emissions which result from vehicles, animal were inhalated once daily and for (24) day continuously .Untreated group (control group) were inhaleted filtered air. emission exhaust from motor vehicles(4) .Vehicle exhaust from benzene emission is a widespread air pollutant ;these exhaust which result from vehicles may cause different type of toxicities resulting in different pathologies(5). Benzene is among the most important air pollutants, which associated with adverse health effect, after exposure to benzene, several factors determine whether harmful health effects will occur, as well as the type and severity of such health effects. These factors include the amount of benzene to which you are exposed and the length of time of the exposure (1). Long term exposure to benzene emission has been associated with increase risk of death from cardiopulmonary diseases (6). Also it has been proved to induce serious pulmonary injury, among been assumed to be mediated by vascular endothelial cell damage (7). The aim of this study to investigate the histopathological changes due to the effects of benzene exhausts emission on some organs in white rat.

Experimental design: - The animals were randomly divided into 2 equal groups, ten animals for each group:

First group: The animals inhalated with filtered air only, this group call (control group).

Second group: The animals inhalated with benzene exhaust emission which result from burning of benzene from internal combustion engines of homes generator for $1 / 4$ hours every day for (24) days. For histopathology, pieces of $1 \mathrm{~cm}^{3}$ from lung ,heart ,liver ,kidney ,spleen and 
testis were taken then kept in $10 \%$ neutral buffered formalin for fixation, processed routinely in histokinette, cut at $5 \mathrm{Mm}$ thickness by rotary microtome and then

\section{Result \&Discussion}

Vehicle motor exhaust contains many toxicants and poses a potential health hazard, benzene exhaust emission may cause toxicological damage in many organs of animals, it suggested that the oxidative damage produced by inhalation may influence or promote the progression or occurrence of some histopathological states of various organs (9).

Lung :- Chronic inflammatory reaction was noticed and characterized by granulomatous tissue surrounding by fibrous connective tissue and infiltration of inflammatory cells such as neutrophils, macrophages which present in the site of lesion .This results agreed with (1);(10), they showed that benzene emission in rat very toxic. Also we showed desquamation of epithelium of bronchi and enlargement of alveoli with ruptured alveolar septa, this condition called pulmonary emphysema and this nearly similar to the results of (11), they reported that benzene emission(carbon monoxide, sulfure dioxide, ozone and nitrogen dioxide) experimentally induced pulmonary emphysema on the adverse health effect in rats with chronic inhalation and high exposure to benzene exhaust emission, also benzene exhaust emission have been proved to induce serious pulmonary injury, among been assumed to be mediated by vascular endothelial cell damage(7)

Heart:-The histopathological changes in the myocardium of treated rats were revealed cardiac muscles suffer extensive coagulative necrosis, and swelling with loss of striations (cloudy swelling). In this aspect, results agree with previous studies ,especially with (12);(9), also they showed that these microscopic changes may be due to cardiovascular impairment.

Liver:-The liver showed marked diffuse vacuolation in hepatocytes , it may be occur due to accumulation of fat as a result of disturbance of fat metabolism due stained with Haematoxylin and Eosin stain then it examined under light microscope(8). to toxic effects of benzene exhaust emission .The above result was reported by (13);(14 ), they reported that benzene exhaust emission(carbon monoxide and sulfur dioxide) cause toxic effect of hepatocyte . Also the results showed hyperplasia of bile duct which considered as compensatory hyperplasia due to impaired function of hepatocytes and hindrance (distortion and lack of blood supply ), necrosis and congestion of central vein occur in liver. The above change could represent an injury to the liver due to toxic effect as it reported by (15).

Kidney :- The kidneys were note effected in all treated animal . It showed hemorrhage atrophy and marked dilatation of convoluted cortical tubules (15);(13) ,reported that the toxic effect which appeared in kidneys because the kidney are the main site of excretion of benzene emission(sulfur dioxide and carbon monoxide) also benzene emission level increase in the body, it causes sever renal toxicity result in dilation of nephrous tubules due to accumulation of benzene exhaust emession in the nephrons.

Spleen: - The histopathological changes were revealed depletion of the white pulp lymphoid tissue and proliferation of red pulp. this result proved by (16);(13 ), they note that long term exposure of benzene exhaust emission(nitrogen dioxide and sulfur dioxide) cause toxicity of lymphoid tissue such as spleen or lymph node, also the spleen play a role in immunity .

Testis :- Microscopic examination of testis showed that there is vaculation of spermatogonia ,evidence of spermatogenesis suppression and reduced number of spermatozoa, it was reported by ( 17);(15);(18 ) .The changes appeared due to long term of exposure to benzene exhaust emission(sulfur dioxide and carbon monoxide). Epididymal tubules of 
treated groups are empty and it not contain sperms this reported by (13), they indicate

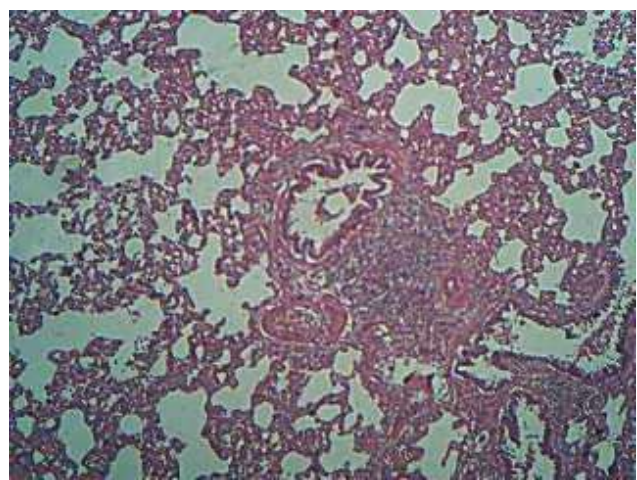

Figure (1):lung.(second group), chronic Inflammatory reaction characterize by Granulomatous tissue \&infiltration of Inflammatory cells.50XH\&E.

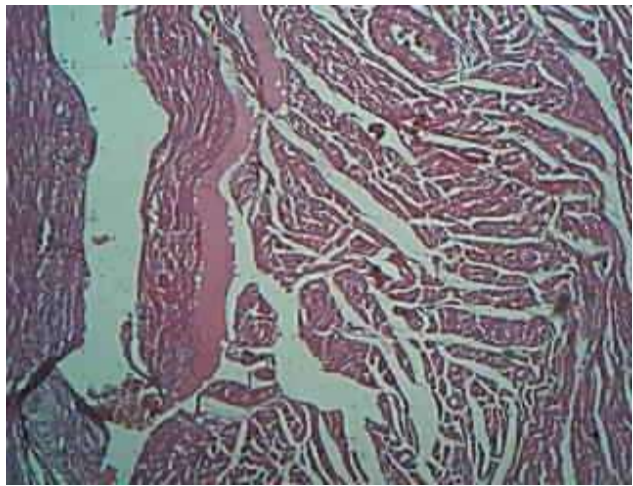

Figure (3):Lung.(second group), congestion and infiltration of inflammatory Cell.50XH\&E.

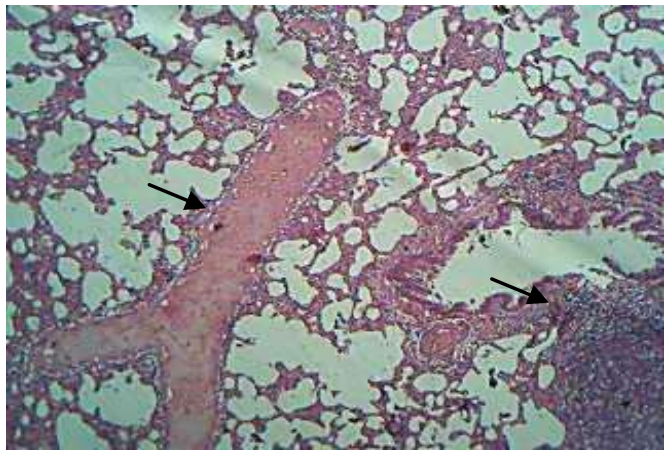

Figure (5): Heart. (Second group), Cloudy swelling of cardiac muscle. 50XH\&E. the benzene exhaust emission cause sever toxicity of reproductive system.

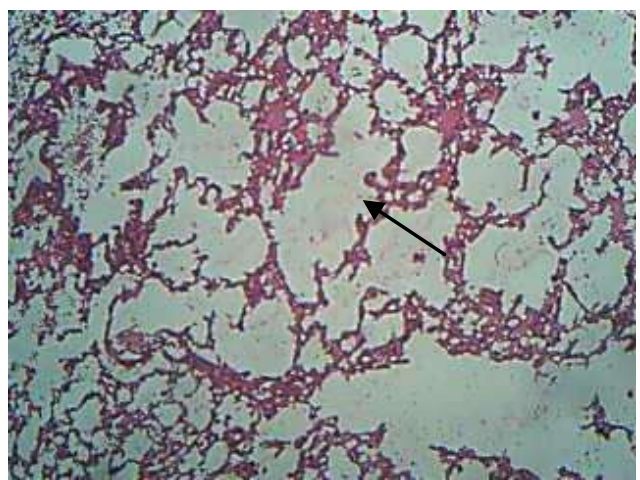

figure(2):lung.(second group), enlargement of alveoli with rupture of alveolar septa (pulmonary emphysema).50XH\&E.

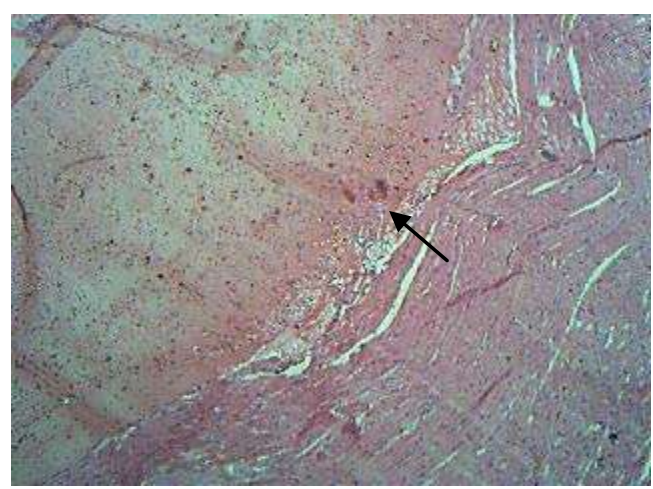

figure (4):heart..(second group) extensive necrotic area in cardiac muscle.50XH\&E.

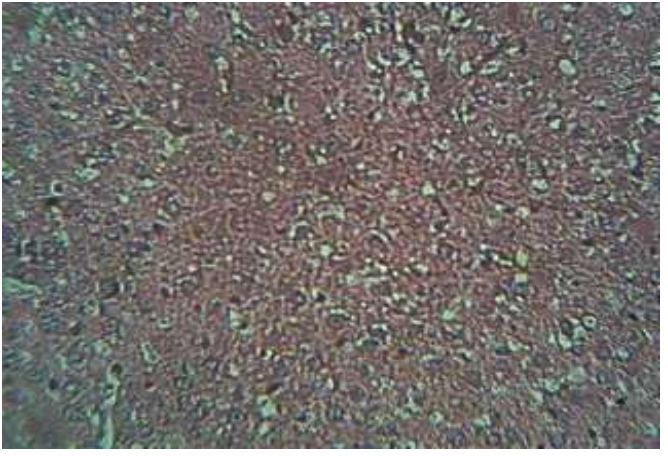

Figure (6): liver. (Second group), Marked diffuse vacuolation of hepatocyte.50XH\&E. 


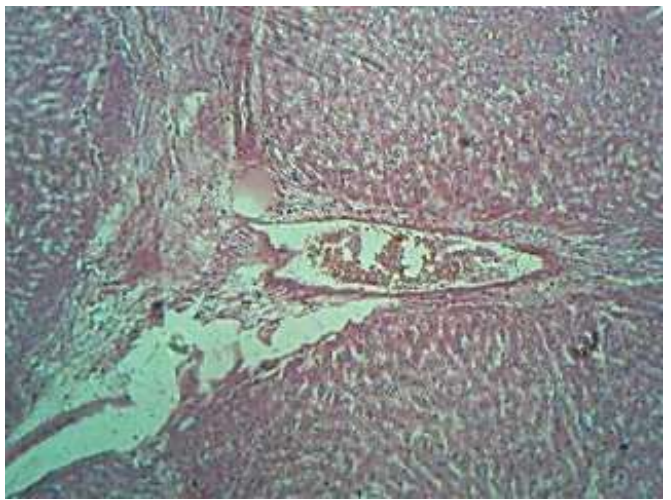

Figure (7):liver. (Second group), hyperplasia of bile duct and congestion of central vein.50XH\&E.

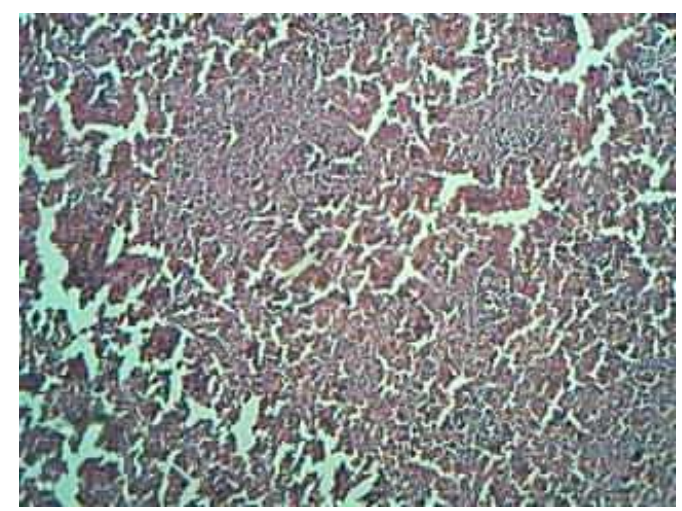

Figure (9):Spleen.(second group), depletion of the white pulp lymphoid tissue . 50XH\&E.

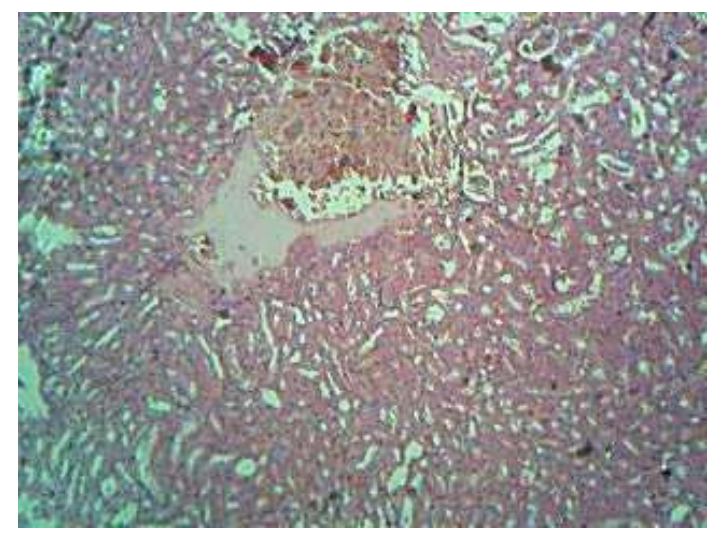

Figure (11):Kidney.(second group), hemorrhage in convoluted cortical tubules.50XH\&E.

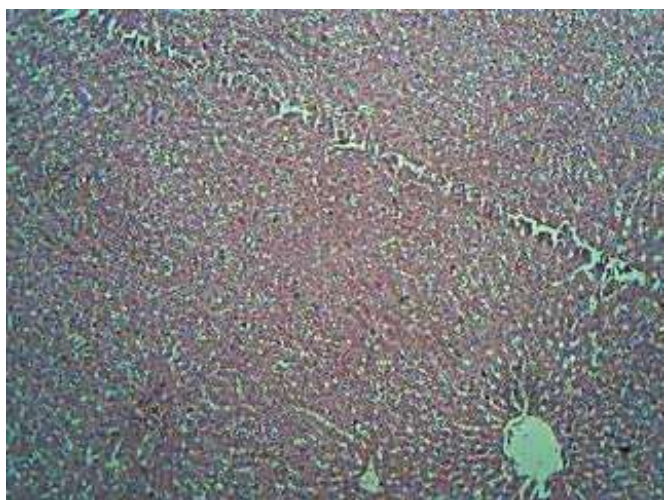

figure (8):liver. (Second group), necrosis of hepatocyte.50XH\&E.

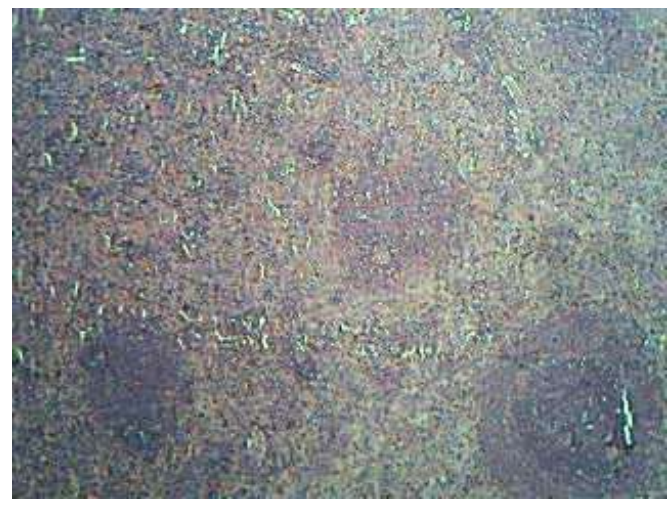

Figure(10):Spleen.(second group), proliferation of red pulp.200XH\&E.

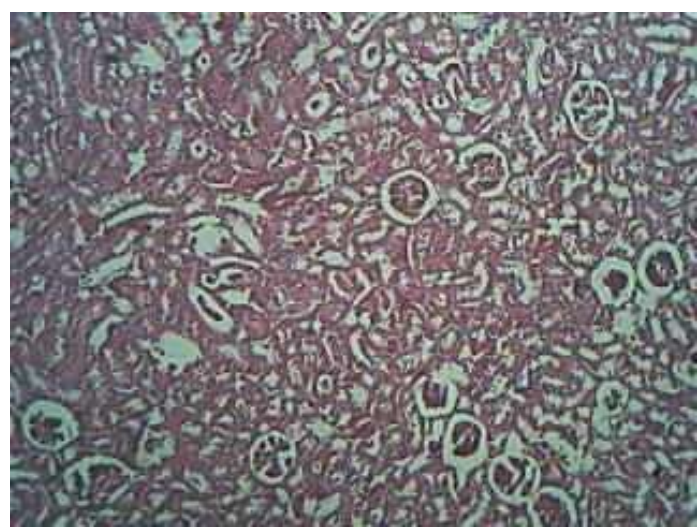

Figure (12):kidney.(second group), atrophy and marked dilatation of convoluted cortical tubules.50XH\&E. 


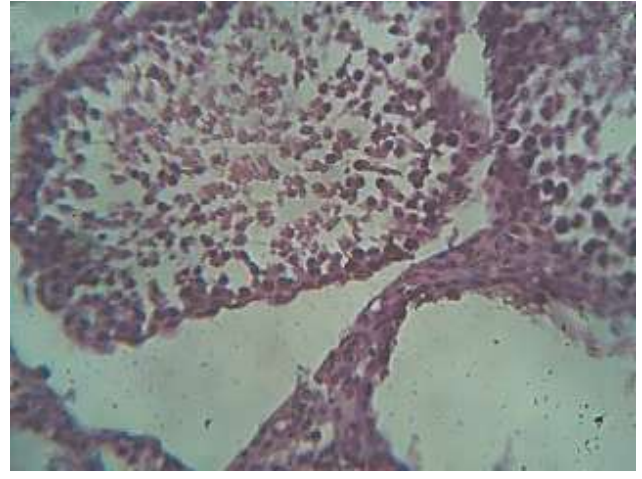

Figure(13):testis.(second group), evidence of suppression spermatogenesis. $50 X H \& E$.

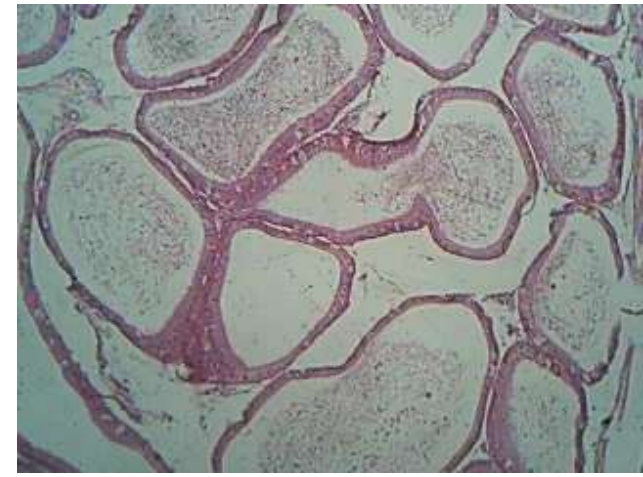

figure( 14):epididymus.(second group),

Reduse number of spermatozoa and epididymal tubules empty.50XH\&E.

\section{References}

1. Seagrave,J.;McDonald,J.D.;Bedrick,E.; Edgerton, E.S.;Gigliotti, A.P.; Jansen, J.J.;Kel, L.;Naeher, L.P.; Seilkop, S.K.; Zheng, M. and Mauderly, J.L.(2006) .Lung toxicity of ambient particulate matter from southeastern U.S.sites with different contributing sources :relation ships between composition and effects .114(9):1387-1393.

2. Rae,P.S.;Ansari,M.F.;Gavane,A.G.;Pan dit,V.L.;Nema,P. and Devotta,S. (2007). Seasonal varuation of toxic benzene emission in petroleume refinery.128(1-3): 323-328.

3. Schwela,D. (2000). Air pollution and health in urban areas .Environ Health .Jan-Jun;15(1-2):13-42.

4. Koren,H.S.(1995).Associations between criteria air pollutants and asthma .103.Suppl.6:235-242.

5. Sohail,E;Aqeela, I.;Sidra,A.; Fatime, B.; Muhammad,A.; Muhammad, N.; Chae,W.and Bumseoke,K. (2009). Toxicological evaluation of the effects of 2-stroke autorick shaw smoke solutions on wound healing .Enviro. Toxicol. and Pharma. Vol(27); issue. (3): 373-383.

6. Chen,L.C. and Hwang, J.S.(2005). Effect of subchronic exposures to concentrated ambient particles (CAPs) in mice-
IU.characterization of acute and chronic effects of ambint air fine particulate matter exposures on heart -rate variability .Inhal. Toxicol. 17(4-5):209-216.

7. Bai,Y.; Suzuki,A.K.and Sagai ,M. (2001) .The cytotoxic effect of diesel exhaust particles on human pulmonary arteries role of active oxygen species .Free Radic.Biol. Med. 30(5):555-562.

8. Luna, L.G.(1968). Manual of histologic staining methods of the armed forces .Institute of pathology $.3^{\text {rd }}$ Ed.,Mc Graw-Hill Book Company ,N.Y., Toronto , London,Sydney;pp:12-31.

9. Ziqiang, M.(2003). Oxidative damage of sulfure dioxid on various organs of mice :sulfur dioxide is a systemic oxidative damage agent .Inhalation Toxicology .vol.(5),no.(2):181-195.

10. Harkema,J.R.;Keeler,G.;Wanger,J.;Mo rishita,M.;Timm,E.;Hotchkiss,J.; Marsik, F.; Dvonch, T. and Kaminski, N. (2004). Effects of concentrated ambient particles on normal and hypersecretore airways in rats. 120:1-68.

11. Mauderly,J.L.; Bice,.E.; Cheng,Y.S.; Gillett,N.A.; Henderson,R.F.; Pickrell, J.A. and Wolff, R.K. (1989).Influence of experimental pulmonary emphysema on the toxicological effects from 
inhaled nitrogen dioxide and diesel exhaust. Res.Rep. Health. Eff. Inset. 30:1-47.

12. Upadhyay,S.;Stoeger,T.;Harder,V.;Tho mas,R.F.;Schladweiler,M.C.;Sem mlerBehnke,M.;Takenaka,S.;Kar q,E.;Reitmeir,P.;Bader,M.;Stamp fl,A.;Kodavant,U.P.and Schuiz, H. (2008) .Exposure to ultrafine carbon particles at levels below detectable pulmonary inflammation affects cardiovascular performance in spontaneously hypertensive rats. part Fibre Toxicol .5:19.

13. Ziqiang,M.;Guohua,Q.;Bo, Z. (2005). DNAdamage in mice treated with sulfure dioxide by inhalation .vol(46);issue(3);p:(150-155).

14. Srivastava,P.K.; $\quad$ Chaudhary,V.K.; Chaunhan,S.S.; Srivastava, V.K. and Misra,U.K. (1985). Biochemical and pathological effects of fly ash on lung ,liver,and bllod.vol (13) ;no . (4);p:(441-452).

15. Medinsky,M.A.;Wolf,D.C.;Cattley,R.C ;Wong,B.;Janszen,D.B.;Farris,G. M.;Wright,G.A.and Bond, J.A. (2006) .Effects of athirteen - week inhalation exposure to ethyltertiary butyl ether of ficher -344 rats and CD-1 mice .Toxicol.sci.5(1):108-118.

16. Azoulay,D.E.;Gougerot,P.M.;Kraus,L. and Moreanu,J. (1987).In vivo nitrogen dioxide exposure depresses spleen cell in vitro mitogenic responses:effects of sulfur compounds .EnvironRes.42(1):130-139.

17. Huang,J.Y.;Liao,J.W.;Liu,Y.C.;Lu,S.Y ;Chou,C.P.;Chan,W.H.;Chen,S.U and Ueng, T.H. (2008). Motorcycle exhaust induces reproductive toxicity and testicular interleukine -6 in male rats .Toxicol. Sci. 103(1):137148.

18. Gunnison ,A.F.;Sellakumar,A.;Currie ,D. and Snyder ,E.A. (1987). Distribution,metabolism and toxicity of inhaled sulfur dioxide and endogenously generated sulfite in the respiratory tract of normal and sulfite oxidase deficient rats. Journal of Toxicology and Environmental Health. $\quad \mathrm{P}(\mathrm{A}) ; \operatorname{vol}(21) ; \quad$ issue (1\&2): 141-162.

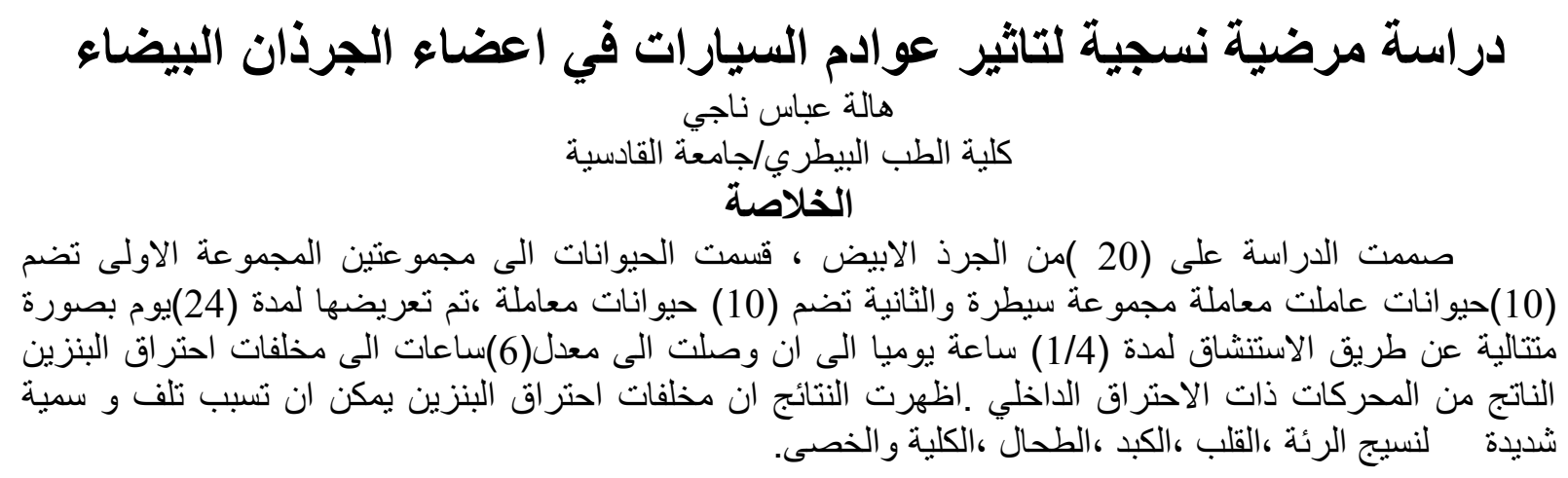

